Mots. Les langages du politique

\title{
Du discours politique au discours expert : le changement politique mis hors débat?
}

Roser Cussó et Corinne Gobin

\section{OpenEdition}

Journals

Édition électronique

URL : https://journals.openedition.org/mots/14203

DOI : $10.4000 /$ mots. 14203

ISSN : 1960-6001

Éditeur

ENS Éditions

Édition imprimée

Date de publication : 1 novembre 2008

Pagination : 5-11

ISBN : 978-2-84788-147-9

ISSN : 0243-6450

Référence électronique

Roser Cussó et Corinne Gobin, «Du discours politique au discours expert : le changement politique mis hors débat? », Mots. Les langages du politique [En ligne], 88 | 2008, mis en ligne le 01 novembre 2010, consulté le 23 avril 2022. URL : http://journals.openedition.org/mots/14203 ; DOI : https:// doi.org/10.4000/mots. 14203 


\section{Du discours politique au discours expert. Le changement politique mis hors débat?}

Il semble difficile d'ignorer la tendance à une profonde homogénéisation du discours politique institutionnalisé à laquelle nous assistons ces deux dernières décennies. Cette homogénéisation résulte, pour partie, d’une circulation lexicale intense de lexèmes ou de mots à consonance «technique». Nous retrouvons ainsi les mêmes expressions perçues comme des catégories de description de la réalité dans la bouche d'hommes et de femmes politiques, toutes tendances confondues, de banquiers, de professeurs d'université, de syndicalistes... Les mêmes mots et expressions servent à tous les niveaux de l'exercice du pouvoir politique : enceinte des organisations internationales, conclaves ministériels nationaux, institutions régionales, réunions municipales. Ils composent également le stock lexical banal utilisé par les médias quotidiennement pour commenter la vie politique et sociale. Citonsen quelques-uns: Formation tout au long de la vie, taux d'emploi, taux de stabilité, stock de capital humain, coûts salariaux, assainissement des finances publiques, vieillissement démographique, politique d'emploi active, employabilité des travailleurs.

Ayant regroupé, par un travail collectif, cent trente de ces mots et /ou syntagmes dans un abécédaire critique des nouveaux mots du pouvoir, Pascal Durand les assimile «[...] à la régression consentie de la politique à une technologie de la corporate governance et à l'application généralisée de la logique de l'économie de marché (convertie en lois de l'économie)» (Durand, 2007).

L'usage de ces mots et formules était, ily a vingt ans à peine, beaucoup plus marginal. Il est possible de constater cette évolution en travaillant dans une perspective diachronique sur lexique politique à l'intérieur de grands corpus politiques chronologiques (Gobin, Deroubaix, 1994; Deroubaix, 1996).

S’il semble évident, par l'expérience de telles études, que de nouveaux mots ou expressions sont créés régulièrement par le monde politique pour répondre aux questions de l'heure et qu'il est ainsi juste de voir le stock lexical politique de gouvernement d'une société comme un sablier qui se dévide vers le passé et se remplit par le présent, il y a néanmoins des périodes où 
un bouleversement rapide et marqué du lexique correspond à un changement dans l'exercice même du pouvoir politique.

Nous sommes, depuis les années quatre-vingt, dans un contexte progressif mais intensif de transnationalisation et de concentration de la décision politique, à travers le poids qu'ont pris à la fois des organismes de pouvoirs collégiaux (G8, Union européenne), et les organisations économiques internationales (Banque mondiale, Fonds monétaire international, Organisation mondiale du commerce...). Ce déplacement de la production du lieu central du programme et du discours matriciels de référence, de la sphère nationale vers des sphères technocratiques, car peu soumises au débat contradictoire, semble déboucher sur la production d'un imaginaire collectif internationalisé dominant qui n'est pas présenté comme un discours politisé et partisan, mais comme un discours neutre.

C'est ce discours politique de «bon sens général », commun à de très nombreux acteurs parce qu'ils veulent être acteurs et reconnus comme tels par ces nouveaux lieux de pouvoir, que nous qualifions de discours expert.

\section{Les principales caractéristiques du discours expert}

Une des premières caractéristiques de ce registre de discours est qu'il est produit par des organismes qui ancrent une bonne part de leur légitimation justement sur la capacité d'expertise qui leur est attribuée. Bernard Maris fait à ce titre une utile distinction entre le discours savant qui construit sa légitimité par la confrontation et le débat scientifiques entre pairs et le discours expert qui impose sa légitimité par la relation particulière qui s'est établie entre ces experts, les pouvoirs politiques et l'opinion publique.

«L'expert n'a aucune justification savante [...]. Il est promu par les médias ou les politiques mais ne subit plus aucun contrôle, puisque ni l'opinion, ni le pouvoir n'ont la compétence nécessaire.» (Maris, 2002, p. 117)

En deuxième lieu, ce discours revêt les oripeaux d'une apparente neutralité politique associant subtilement des codes de type technique - recours important, dans ces discours, à de nombreux indicateurs statistiques comme éléments de preuve mais aussi comme objectifs politiques (Conter, 2008) - à un vocabulaire flou, abstrait, naturalisant qui aseptise en quelque sorte toute velléité polémiste. La force de ce discours expert est en effet qu'il se donne l'apparence d'une simple correspondance au juste état des choses.

En troisième lieu, ces discours se présentent comme dépolitisés, via la neutralisation de mots qui semblent être "passe-partout», convaincants pour tous car compréhensibles a priori par tous. Ce processus de dépolitisation du discours politique opérée par des groupes dominants dans le champ économique et politique n'est pas neuf. Pierre Bourdieu l'avait déjà épinglé en 1982 
dans Ce que parler veut dire: « [...] ayant intérêt au laisser-faire, ils [les dominants] travaillent à annuler la politique dans un discours politique dépolitisé, produit d'un travail de neutralisation ou, mieux, de dénégation, qui vise à restaurer l'état d'innocence originaire de la doxa et qui, étant orienté vers la naturalisation de l'ordre social, emprunte toujours le langage de la nature» (Bourdieu, 1982, p. 155).

Ce qui est neuf par contre, et qui constitue une quatrième caractéristique contemporaine, concerne cette forte homogénéisation lexicale. Celle-ci peut être comprise comme l'entrecroisement de phénomènes multiples: l'effet transnationalisant de la mondialisation économique, la banalisation et le renforcement du capitalisme qui naturalise l'économie de marché, le renouvellement lexical intense (et traduit dans 23 langues!) opéré par l'Union européenne pour susciter l'adhésion à l'Union économique et monétaire (Gobin, 2002), la concentration des médias qui aboutit à un appauvrissement des lieux du débat contradictoire.

\section{Une structure argumentative fortement marquée}

Mais au-delà de mots nouveaux, la structure argumentative du discours expert est aussi particulière. L'heure n'est plus aux sentiments ni aux utopies militantes. La conviction, l'adhésion du public est toujours recherchée, mais non à travers la présentation d'un programme politique de transformation ou de conservation sociale. Les arguments fuient le conflictuel, se cantonnent dans le banal et dans le rassurant; si ces discours sont en effet capables d'énoncer (d'annoncer?) les problèmes - toujours graves - du siècle, ils peuvent tout aussitôt énumérer les solutions pour y faire face. De multiples indicateurs techniques sont mobilisés pour montrer que la force de l'argument repose sur du concret, du chiffre, du vrai... et donc de l'indiscutable. L'approche descriptive du réel est quasi clinique: diagnostic et remède, avec une seule explication possible dans la détermination des problèmes et une seule voie pour y remédier. Il ne s'agit pas de programmation politique à proprement parler car la voie à emprunter serait naturelle. Le discours expert tire, en effet, sa légitimité de sa grande capacité à pouvoir paraphraser le réel: non pas apte à lire dans les méandres de l'économie les auspices d'un monde incertain, mais capable plutôt de décrypter, de comprendre et donc d'accompagner les mouvements présents et naturels des mutations sociétales. La société, entité qui devient très abstraite dans ce cadre, saurait d'elle-même comment s'adapter aux mouvements de la vie: le discours expert mettrait juste en mots cette capacité d'auto-adaptation. Dès lors, souvent objectifs et solutions se confondent au sein d'une rhétorique auto-réalisante et circulaire: donnons cet exemple, tiré de nos analyses 
des textes produits par l'Union européenne, où, dans la rhétorique sur la politique d'emploi, il est impossible de déterminer s'il faut plus de croissance pour favoriser la création d'emplois ou créer de l'emploi pour booster la croissance (Gobin, 2005).

\section{Des émetteurs situés dans la sphère internationale et transnationale}

Les organisations internationales et européennes (Banque mondiale, Organisation de coopération et de développement économiques, Union européenne), ont été au cœur de cette transformation lexicale et argumentative depuis le début des années 1980. Alors que le changement politique, dans une conception démocratique de la souveraineté nationale, était auparavant intrinsèquement lié à l'idée de conflits légitimes autour de programmes politiques divergents, aujourd'hui, il se réalise progressivement par le biais de rapports techniques qui égrènent, venus d'en haut, les réformes techniques que les gouvernements doivent appliquer par nécessité, juste pour correspondre à la réalité des choses. Les organisations internationales et l'Union européenne banalisent ainsi le changement, tentant de le mettre au-delà des tensions politiques, au-delà du débat politique conflictuel... Les questions politiques de l'heure sont travesties en questions techniques, rationnelles, présentées comme susceptibles de dépasser tout clivage politique.

À l'ère de la souveraineté nationale, les rapports politiques entre gouvernements nationaux et organisations internationales semblaient clairs: ceuxlà mandataient celles-ci pour que leur soient transmis des avis et des rapports comme autant de commandes permettant de faire le point sur une question avant d'ouvrir un débat (principalement parlementaire) préalable à la prise de position ou de décision collective.

À l'ère de la mondialisation, les États reçoivent des rapports qui leur enjoignent de mettre en ordre leur politique nationale qui serait en inadéquation avec le cours des choses.

Cette situation invite à explorer, par l'analyse de ce profond renouvellement lexical, les changements à l'œuvre dans les rapports entre politique et expertise. Que dit d'autre Guy Hermet quand il se demande si le terme de gouvernance n'est pas le nom de «l'après-démocratie» (Hermet, 2005), ou quand Fritz Scharpf théorise la spécificité du mode de gouverner de l'UE comme une «légitimation par les outputs», s'éloignant ainsi de la légitimité formalisée des modes de contrôle démocratiques (Scharpf, 2000)?

Ainsi, entre l'expert et le politique, les frontières se brouillent: n'est-ce pas parce que la légitimité politique issue du suffrage universel s'est dévalorisée auprès des dirigeants? 


\section{Style de rhétorique et programmes d'action}

Le dossier coordonné par Gilbert Rist sur Les mots du pouvoir. Sens et nonsens de la rhétorique internationale (Rist, 2002) avait mis l'accent sur le style de la rhétorique internationale qualifiée de "style formulaire», les discours produits dans les rapports des organisations internationales semblant être construits sur un enchainement de formules stéréotypées et généralisantes générant une impression globale de vacuité du dire. Mais au-delà de l'impression d'être submergé par des mots creux, se manifestait la volonté des auteurs de présenter ces textes comme autant de programmes qui orientent l'action politique, autant par le déphasage du réel qui s'y opère que par ce qui n'y est pas dit.

Le dossier présenté dans le présent numéro s'inscrit dans le prolongement du chantier ouvert par Gilbert Rist. Deux articles, celui de Jean-Louis Siroux et celui de Francine Mestrum, reviennent sur la question de la rhétorique à l'œuvre dans les grandes organisations internationales. À ceci s'ajoute un article consacré à l'analyse du discours de l'Union européenne par Roser Cussó. Ce discours est construit aujourd'hui en filiation avec celui porté par les organisations internationales: s'y trouve le même projet global de produire un sentiment d'urgence et de nécessité à des réformes fondamentales, qui touche le cœur du fonctionnement des anciennes institutions démocratiques, en y exportant la pensée monétariste et de promotion du libre-échange comme approches hégémoniques. Y circulent les mêmes expressions figées, le même vocable dont un échantillon a été donné en ouverture de cette introduction. In fine, au bout du processus de réformes, c'est l'idée même de l'État et de l'action publique qui sort radicalement modifiée, à travers une diminution de sa capacité redistributive et une diminution du contrôle des acteurs sociopolitiques nationaux (dont les organisations syndicales) sur le contenu des politiques de réforme qui sont à l'œuvre (Gobin, 2006).

Ces trois analyses se focalisent sur le discours d'organisations internationales clés, détentrices d'une autorité politique certaine.

Jean-Louis Siroux s'attache à décrire, tant sur le plan du contenu lexical que de la forme argumentative, l'introduction des rapports annuels rédigés par l'OMC. Il examine quelles sont les formules de rhétorique ainsi que le lexique mobilisés qui en font une sorte d'archétype de «discours politique dépolitisé». Son article se prolonge en un questionnement sur la façon dont un acteur important peut construire un «dire» légitime et crédible à partir de la production de textes pourtant bien peu «brillants».

Françoise Mestrum s'interroge sur la surmobilisation que fait la Banque mondiale du thème de la pauvreté dans ses publications et rapports récents. À travers une analyse systématique du lexique et des arguments mobilisés, 
elle y voit une transformation radicale de la notion de «développement» qui va permettre de légitimer l'idéologie du libre-échange néolibéral. Par un discours général de la lutte contre la pauvreté via l'application de la bonne gouvernance, c'est l'ensemble des rapports à l'État et à la citoyenneté politique et sociale que la Banque mondiale retravaille en faveur de la promotion d'une nouvelle utopie libérale de l'harmonie entre riches et pauvres, unis, pour ceux qui en ont la capacité, par «l'audace du risque», apanage de l'individu libre et responsable.

Roser Cussó analyse l'environnement lexical des textes européens qui décrivent la nécessité de l'avènement de l'Union européenne comme économie de la connaissance. L'analyse de contenu du contexte des mots marqués par les plus hautes fréquences révèle un argumentaire qui, au nom de la révolution de la mondialisation, ordonne une réforme profonde des systèmes éducatifs et des compétences individuelles afin d'aboutir à la mise en place d'un marché européen de l'emploi. Cette réforme serait aussi une illustration du nouveau modèle de la régulation publique à promouvoir, où l'autorité publique devient plus un «intermédiaire » parmi d'autres que le décideur.

Plusieurs éléments se font écho dans l'analyse offerte par ces trois articles de discours institutionnels internationaux: on y trouve un discours politique expert qui commande des réformes profondes aux États, réformes présentées non comme des choix de société dont il faudrait débattre mais comme des impératifs techniques qui correspondraient à la marche «naturelle» de l'économie ; les acteurs-clé du politique (États, partis politiques), sont ou dénigrés ou absents, ou encore soumis à réforme pour rompre avec leur rôle passé; un nouveau modèle de la vie en société et du contenu de l'action publique est proposé, mettant en valeur l'initiative privée.

Enfin, un quatrième article ouvre un champ original d'investigation scientifique en s'interrogeant sur la capacité d'autonomie lexicale qu'il reste à des acteurs plongés dans ces grands systèmes institutionnels internationaux. Ainsi, Anne-Catherine Wagner réfléchit sur ce qui reste de possibilités, aujourd'hui, pour les militants syndicaux qui œuvrent à l'échelon de l'Union européenne, d'être en dissonance lexicale avec le discours expert européen largement repris par ailleurs dans les textes de résolutions de la Confédération européenne des syndicats. Son travail de décorticage lexical s'opère à partir d'entretiens.

\section{Références}

Bourdieu Pierre, 1982, Ce que parlerveut dire, Paris, Minuit.

CONTER Bernard et al., 2008, "Ombres et lumières sur l'emploi et les salaires: le rôle des indicateurs statistiques européens », R. Cussó et al. éd., Le conflit social éludé, Louvain-la-Neuve, Academia Bruylant, p. 119-140. 
Deroubaix Jean-Claude, 1996, Les déclarations gouvernementales en Belgique (19441992). Étude de lexicométrie politique, thèse de doctorat en sciences du langage, Université de la Sorbonne nouvelle, Paris 3.

Deroubaix Jean-Claude, Gobin Corinne, 1994, Quand la Commission se présente devant le Parlement. Étude du vocabulaire des discours de présentation de la Haute Autorité de la CECA et de la Commission de la Communauté européenne devant le Parlement européen (1952-1993), Bruxelles, Recherches européennes en sciences humaines.

DURAND Pascal éd., 2007, Les nouveauxmots du pouvoir. Abécédaire critique, Bruxelles, Aden.

GobIN Corinne, 2002, "Le discours programmatique de l'Union européenne. D'une privatisation de l'économie à une privatisation du politique? ", Sciences de la société, $n^{\circ} 55$, p. $156-169$.

- 2005, «L'Union européenne : où est passé l'acteur?», Cahiers lillois d'économie et de sociologie, p. 65-88.

- 2006, «La transformation du social au cœur de la politique publique de l'Union européenne », Revue de l'Institut de sociologie, n 1-2, A. Van Haecht éd., Éducation et formation. Les enjeux politiques des rhétoriques internationales, p. 39-56.

HERMET Guy, 2005, «La gouvernance serait-elle le nom de l'après-démocratie? » G. Hermet et al. éd., La gouvernance. Un concept et ses applications, Paris, Karthala, p. 17-47.

MARIS Bernard, 2002, "Légitimation, autolégitimation, discours expert et discours savant», Sciences de la société, $n^{\circ} 55$.

RIST Gilbert éd., 2002, Nouveaux Cahiers de l'IUED, $\mathrm{n}^{\circ} 13$, Les mots du pouvoir. Sens et non-sens de la rhétorique internationale, Paris, Genève, PUF (Enjeux).

Scharpf Fritz, 2000, Gouverner l'Europe, Paris, Presses de Sciences Po, p. 23-30. 